\title{
A Tool for Automatic Enterprise Architecture Modeling
}

\author{
Markus Buschle, Hannes Holm, Teodor Sommestad, \\ Mathias Ekstedt, and Khurram Shahzad \\ Industrial Information and Control Systems, KTH Royal Institute of Technology, \\ Osquldas v. 12, SE-10044 Stockholm, Sweden \\ \{markusb, hannesh, teodors, mathiase, khurrams\}@ics.kth.se
}

\begin{abstract}
Enterprise Architecture is an approach which aims to provide decision support based on organization-wide models. The creation of these models is however cumbersome as multiple aspects of an organization need to be considered. The Enterprise Architecture approach would be significantly less demanding if data used to create the models could be collected automatically.

This paper illustrates how a vulnerability scanner can be utilized for data collection in order to automatically create Enterprise Architecture models, especially covering infrastructure aspects. We show how this approach can be realized by extending an earlier presented Enterprise Architecture tool. An example is provided through a case study applying the tool on a real network.
\end{abstract}

Keywords: Enterprise Architecture, Automatic data collection, Automatic instantiation, Software tool, Security Analysis.

\section{Introduction}

Enterprise Architecture (EA) is a comprehensive approach for management and decision-making based on models of the organization and its information systems. An enterprise is typically described through dimensions such as Business, Application, Technology and Information. [12. These pictographic descriptions are used for system-quality analysis to provide valuable support for IT and business decision-making [6].

As these models are intended to provide reliable decision support it is imperative that they capture all the aspects of an organization which are of relevance. Thus, they often grow very large and contain several thousands of entities and an even larger number of relationships in between them. The creation of such large models is both time and cost consuming, as lots of stakeholders are involved and many different pieces of information have to be gathered. During the creation process the EA models are also likely to become (partly) outdated [1]. Thus, in order to provide the best possible decision support it needs to be ensured that EA models both are holistic and reflect the organizations current state. 
Automatic data collection and model creation would be useful as this would decrease the modeling effort and increase the quality of the collected data.

In this paper we present how the Enterprise Architecture Analysis Tool 6] has been extended in order to automatically instantiate elements in EA models based on results from network scans. In comparison to other tools our implementation focuses on the Application and Technology layer of the organization. This information is gathered through an application of a vulnerability scanner that evaluates the network structure of an enterprise. Thereby attached network hosts and the functionality they provide can be discovered. Another difference is that the presented tool uses EA models for system-quality analysis, whereas commercial applications focus on modeling. As a running example we illustrate how a meta-model designed for cyber security analysis [16] can be (partly) automatically instantiated. The presented implementation is generic and can be used to support any kind of EA analysis.

The remainder of this paper is structured as follows. Related work is discussed in section two. Section three describes the components used to realize the implementation and introduces into the meta-model that is used as running example. Section four describes how the information, which was automatically collected, is used to instantiate the meta-model for security evaluation. Section five exemplifies the tool application on real data collected by scanning a computer network used for security exercises. In section six the presented tool and the underlying approach are discusses as well as future work is described. Finally section seven concludes the paper.

\section{Related Works}

In the EA community there are few initiatives focusing on the data collection process for model instantiation and maintenance. Among the most well-known frameworks data collection is almost completely left to the modeler to handle. There are a couple of tool vendors providing some support. However, it is required in most cases that the needed information is available somewhere else, implying that the data must be collected by someone at some point in time. In the academic EA community most researchers have put their focus on deriving principles and designing methods for model creation and maintenance. Few have implemented their ideas in working software tools. None claims to have the focus on automatic data collection.

\subsection{EA Frameworks}

There are many frameworks covering EA modeling e.g. 2112,19. However, few (none) of these describe and discuss the data collection process used when creating the architecture models. No practical help is presented in these frameworks regarding the data collection for As-is models or for checking the consistencies in continuously updated architecture models (maintaining the architecture). 


\section{$2.2 \quad$ EA Tools}

In current EA tools some approaches addressing automatic data collection can be found. The most common way is to import models that are made in third party software. For example, BizzDesign Architect [3] can import data from office applications and with this data instantiate models. Thereby the automation aspect actually means that data is reused and does not need to be manually entered if it is already available. The interpretation of data documented in the third party software can however be resource- and time consuming, thus contradicting parts of the purpose with automatic data collection.

Other tools, such as for example Troux [20], allow the usage of SQL queries in order to load information from data bases. This approach focuses on the extraction of the data model and process descriptions, thereby the automatic creation of the information architecture as well as the business architecture.

Both approaches assume that the data entered, in the third party applications or data bases, is already available and updated. However, this data still needs to be manually collected in the first place before it can be used.

ARIS Business Architect for SAP [15] supports the reuse and import of SAP process models out of the SAP Solution Manager. Thereby modeling costs are reduced. The SAP process model does only cover certain parts of the complete EA. While other aspects of EA, such as infrastructure, are not considered.

18 presents a software tool and methodology used for collecting architecture information. The tool collects data from project management systems and operational systems, and users can upload documents containing architecture information. This approach thus aims to provide automatic data collection for architecture models. The approach is however still time consuming. Since the tool proposed use .cvs-files the data documentation process still needs to be formalized.

\subsection{EA Methods and Principles}

[5] presents a Wiki-based approach to EA documentation and analysis. According to Buckl et al. companies who start an EA initiative usually do not have a pre-defined information model for this. Many companies start with regular spreadsheets or similar. Instead, Buckl et al. propose a Wiki-based approach for collecting and sorting the information needed for EA management. The main benefit with the Wiki-approach is that the data collection is distributed but still managed by a formal set up. Although the Wiki-based approach proposed seems interesting there is still a need for data collection to provide input to the Wiki.

In 4] an approach for handling change is proposed. The approach is called Living Models and it is based on theories of model based software development and EA management. The idea is to connect IT management, System Operation, and Software Engineering. According to the author three major research challenges have to be met in order to materialize this: 1) Provide a coherent view of the quality status of the systems. 2) Keep track of the quality status as the systems evolve over time. 3) Support the collaboration of stakeholders for 
achieving the necessary quality level. EA models can be used to achieve number one of the three challenges. Automatic data collection would be appropriate for challenge number two. In the paper Breu presents ten principles that are crucial for Living Models. Principle no 2 - Close Coupling of Models and Code states: "Models are generated out of the code (e.g. architecture models)". This would mean automatic generation of models at some architecture levels. Furthermore, Principle no 3 - Bidirectional Information Flow between Models and Code, focus on the idea that information from code can be used to build models as well as information in models can be used to generate code. Throughout the ten principles patterns and meta-model elements are discussed supporting these ideas. However, there is no tool today that can implement and use these principles yet.

9] states that: "Architecture management should produce methodological results in the form of architecture artifacts such as models, standards, etc." Further they state that: "Obviously, it is not possible to assume a consistent EA at a specific point in time, which means there should be a prime focus on dealing with inconsistencies." The authors conclude that architecture models will drift and become inconsistent. They spend some effort on trying to address this problem as it has occurred. What they do not describe is how to collect the information needed to actually compare the real world with the as-is models or update the models with the correct data.

In [2] the focus is on the design of the EA. The main result is a framework for engineering driven EA design and a software tool implementation of this framework. There is no description of the data collection process for the instantiation of models. The design of an EA here basically means deriving the meta-model needed for an enterprise. The software tool implementation proposed is a tool incorporating the framework and meta-model. In [7] the focus is on model maintenance and the main finding reported in this publication is a discussion of the shortcomings of existing model maintenance approaches. The authors present a federated approach to deal with these shortcomings. However, there is no discussion regarding the data collection part of model maintenance.

\subsection{Summary}

There are few initiatives describing and discussing data collection for EA modeling. There are even less initiatives proposing an automatic data collection process. Most research publications related to the topic provide evidence that the topic is important and needs to be addressed.

\section{Preliminaries}

This section describes the three components that were combined in order to automatically create EA models that can be used for security analysis. In subsection 3.1 the vulnerability scanner NeXpose [14, which is used for data collection, is explained. Subsection 3.2 describes the Enterprise Architecture Analysis Tool that is used to generate the models and evaluate them with regards to security aspects. Subsection 3.3 briefly introduces CySeMoL, the used meta-model 
which is partly instantiated using the automated data collection. The overall architecture can be seen in figure 1.

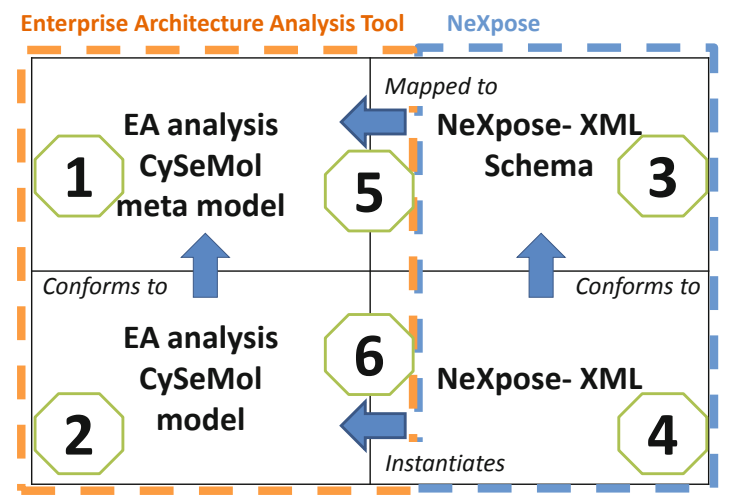

Fig. 1. The used architecture

\section{$3.1 \quad$ NeXpose}

The vulnerability scanner NeXpose was chosen in this project as it has demonstrated good results in previous tests [10. A vulnerability scanner is a software tool which probes a network architecture for various vulnerabilities, for example, poor passwords and software flaws (e.g. unpatched software with known vulnerabilities).

NeXpose [14] is an active (i.e. it queries remote hosts for data) vulnerability scanner capable of both authenticated and unauthenticated scans. Authenticated scans involve providing the scanner with user accounts to hosts. They are typically less disturbing to normal operations and providing a higher degree of accuracy. However, it is not always the case that credentials are readily available for the individual(s) performing a scan.

NeXpose provides information regarding the network architecture in terms of all devices which are communicating over TCP or UDP, e.g. computers, firewalls and printers. The scanner identifies the operating systems or firmware that is running on the scanned devices and any services that are running. If the scanner is given credentials it is also able to assess all applications (and versions thereof) installed on a device and all user/administrator accounts on that device.

More security related functions of the scanner include that it can check for both software flaws and configuration errors. It is also capable of performing web application scans. NeXpose has approximately 53000 current signatures in its engine, with every signature corresponding to a certain vulnerability. NeXpose is also SCAP-compliant [11] and thus compliant with a suite of six commonly used protocols developed by the National Institute of Standards and Technology (NIST): i) Extensible Configuration Checklist Description Format (XCCDF), 
ii) Open Vulnerability and Assessment Language (OVAL), iii) Common Platform Enumeration (CPE), iv) Common Configuration Enumeration (CCE), v) Common Vulnerabilities and Exposures (CVE) and vi) Common Vulnerability Scoring System (CVSS).

\subsection{Enterprise Architecture Analysis Tool}

In 6] we presented a tool for EA analysis. This tool consists of two parts to be used in succession. The first component allows the definition of meta-models to describe a certain system quality of interest (1 in Figure 1). This is done according to the PRM formalism [8] in terms of classes, attributes, and relations between them. Thereafter an execution of the second component is done in order to describe an enterprise as an instantiated model ( 2 in Figure 1), which is compliant to the previously defined meta-model. As the PRM formalism supports the expression of quantified theory the described enterprise can be analyzed with regards to the considered system quality described in the first component.

To use the results gained from NeXpose scans an extension of the tool was necessary. The result of NeXpose's scans can be exported to XML files (4 in Figure 1), which are structured according to a schema definition file (XSD) 1 (3 in Figure 1). We added the possibility to create mappings between XSD files and meta-models ( 5 in Figure 1) in order to automatically instantiate the metamodel based on NeXpose's XML files (6 in Figure 1). The used mapping is discussed in section 4 .

\subsection{CySeMoL}

This paper exemplifies the mapping functionality by instantiating a subset of the meta-model of the Cyber Security Modeling Language (CySeMoL) [17]. This modeling language follows the abstract model presented in [16] and uses the PRM formalism to estimate the value of security attributes from an architecture model. Its meta-model covers both technical and organizational aspects of security and can be seen in Figure 2 (without any attribute relationships shown). CySeMoL includes 22 entities, each with various attributes and relations to other entities. It can require a lot of effort to model a scenario using the CySeMoL as a typical network contains multiple components e.g. computer systems, operating systems, services, password accounts, and application clients. Furthermore, each of these entities have security related aspects (i.e. attribute states) that needs to be modeled in order to achieve satisfactory prediction quality. The states of most of the attributes in the meta-model are defined by the states of their parents. However, the states of some variables need to be defined by the user of the model. For example whether there are vulnerabilities available for an operating system or a service. The required modeling effort could result in errors caused by the individual(s) performing the modeling.

${ }^{1}$ The XSD file (Report_XML_Export_Schema.xsd) is part of the NeXpose Community Edition that can be downloaded from http://www.rapid7.com 
As such, it would be very valuable if parts of the CySeMoL meta-model could be automatically generated - it would save both modeling time and likely increase the accuracy of the resulting prediction model. Four entities, three entity relationships and the states of four attributes can be mapped to elements produced by NeXpose. These concepts, and the mapping as such, are discussed in section 4. While only a subset of the total number of entities and relations could be instantiated, this subset includes entities, relations and attributes which are of high multiplicity in enterprises, and thus require lots of effort to model.

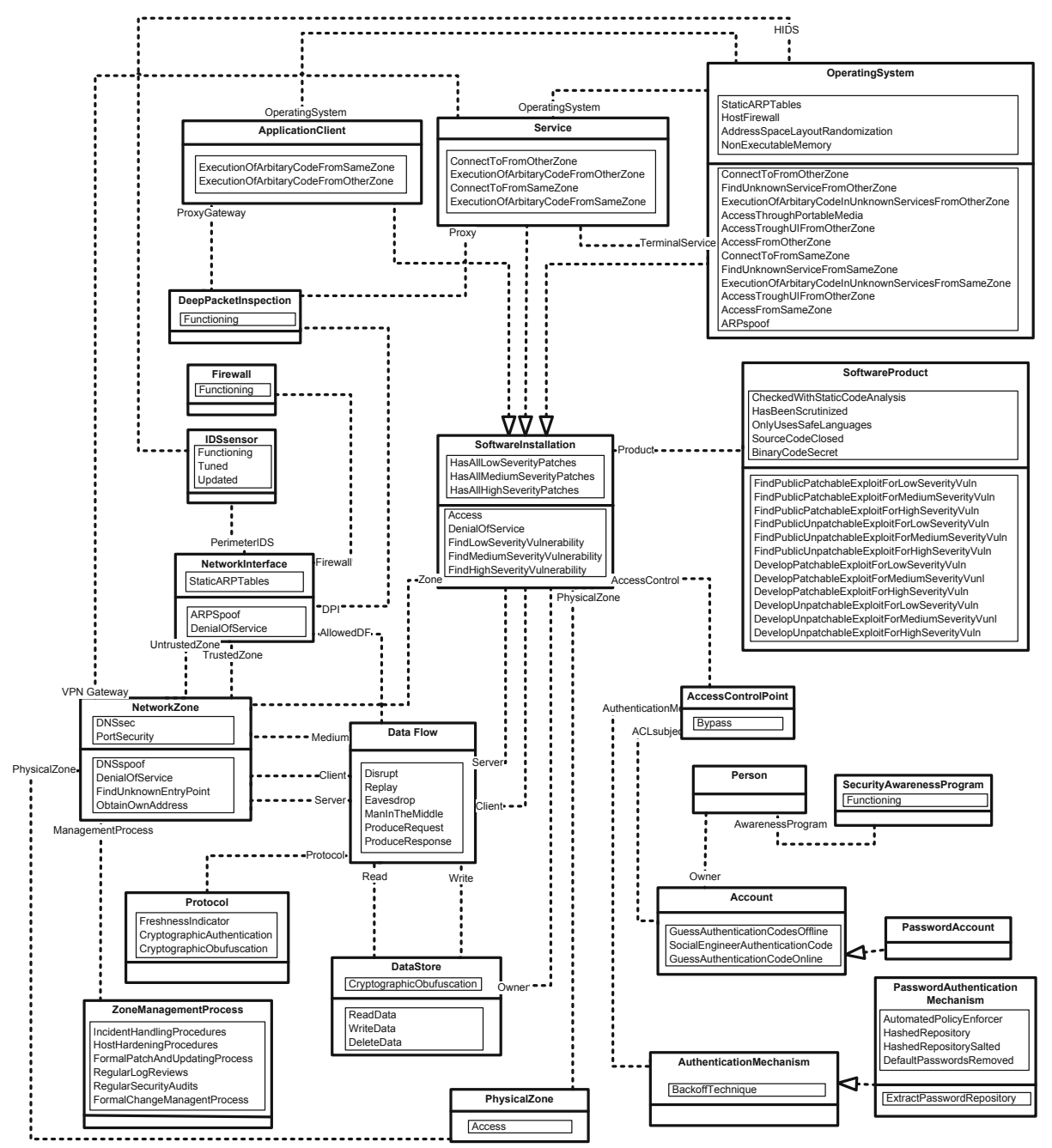

Fig. 2. An overview of $\mathrm{CySeMoL}$ without attribute relations shown 


\section{The Mapping}

This section describes the concepts of CySeMoL that were possible to map to the output of NeXpose, and how these concepts are defined by NeXpose. This mapping was done based on the XSD file that describes the structure of the vulnerability scanning reports produced by NeXpose. Furthermore, the mapping is based on the viewpoint of CySeMoL as this is the focus of the study - to see how much of its contents that can be automatically generated. All of the entities described in this chapter were possible to relate as shown in Figure 2

\subsection{Operating System}

The CySeMoL entity OperatingSystem and three of its attributes are possible to automatically instantiate (cf. Figure 3. These concepts are described below. As can be seen, all information required by CySeMoL regarding these aspects are fulfilled.

OperatingSystem. CySeMoL: The software system which other software is deployed on. Examples include Windows XP SP2 (with a specific set of patches), VMware vSphere 5.1.0 and printer firmwares (and versions thereof). NeXpose: The same definition as CySeMoL, abstractly described as the Operating System of a Node. Gathered information includes e.g. OS type and version, and the IP adress of the system.

It is also possible to gather data for three of the attributes for the OperatingSystem class: Whether the operating system has any low, medium or high severity vulnerabilities as defined by the Common Vulnerability Scoring System 13. (HasAll(Low/Medium/High)SeverityPatches). The CVSS is a quantitative metric on a scale from 0.0-10.0 which grades IT security vulnerabilities according to their criticality. A low severity vulnerability corresponds to 0.0-3.9, a medium severity vulnerability to 4.0-6.9, and a high severity vulnerability to 7.0-10.0. NeXpose detects any vulnerabilities present in the probed operating system(s) and presents them with their corresponding CVSS score.

\subsection{Software Service}

The CySeMoL entity Service and three of its attributes are possible to automatically instantiate (cf. Figure 4. These concepts are described below. As can be seen, all information required by $\mathrm{CySeMoL}$ regarding these aspects are fulfilled.

Service. CySeMoL: A software that functions to service software clients. A Service listens on one or several ports for requests by software clients NeXpose: All relevant overall aspects can be captured, e.g. port number, software name and software version.

It is, as for the OperatingSystem class, also possible to gather data for three of the attributes of the Service class: Whether the service has any low, medium or high severity vulnerabilities (HasAll(Low/Medium/High)SeverityPatches) as defined by the CVSS. NeXpose detects any vulnerabilities present in the probed software service(s) and presents them with their corresponding CVSS score. 


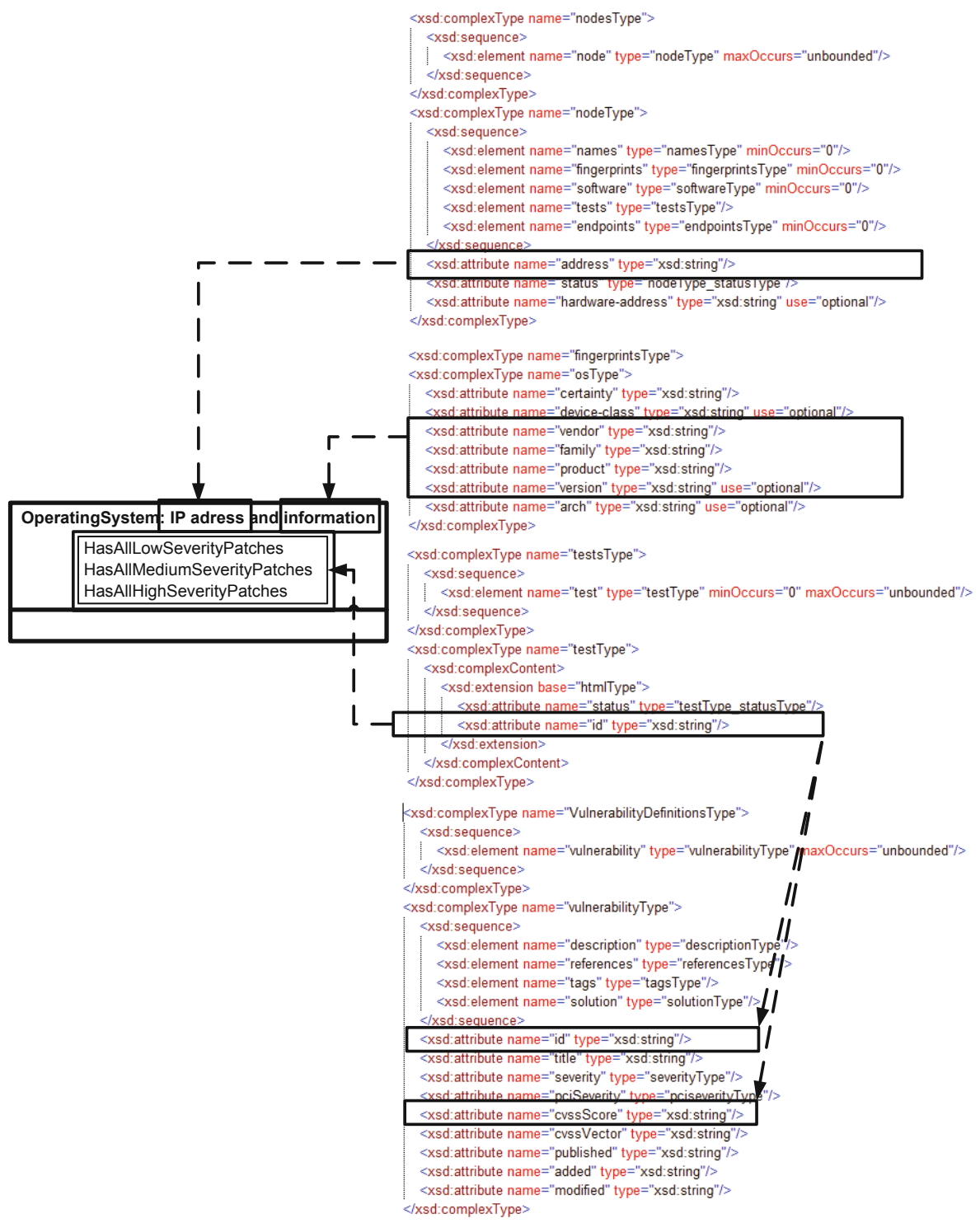

Fig. 3. Mapping of OperatingSystem to NeXpose XML

\subsection{Network Zone}

The CySeMoL entity NetworkZone was possible to map to the output of NeXpose (cf. Figure 5). This is detailed below.

NetworkZone. CySeMoL: A network which enables connected systems to communicate. NeXpose: The IP of each scanned node. As such, it is possible to 


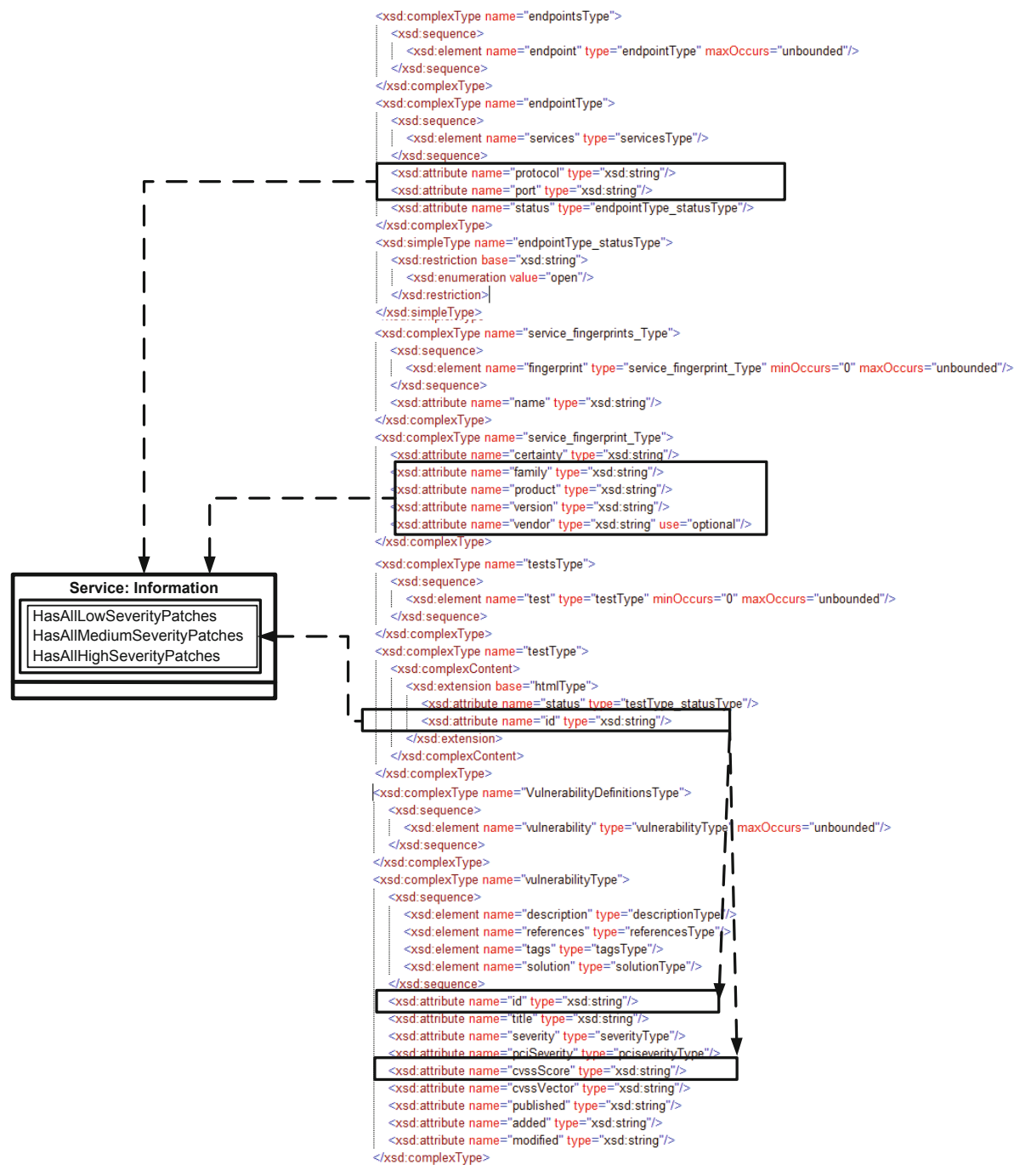

Fig. 4. Mapping of Service to NeXpose XML

group all systems (OperatingSystems) that have IPs on the same subnet (e.g. 172.168.2.x).

\subsection{Software Product}

The CySeMoL entity SoftwareProduct was possible to map to the output of NeXpose (cf. Figure 6). This is described below.

SoftwareProduct. CySeMoL: The type of software. For example, Windows XP SP2 or Apache Webserver. This is also the main difference from a Software- 


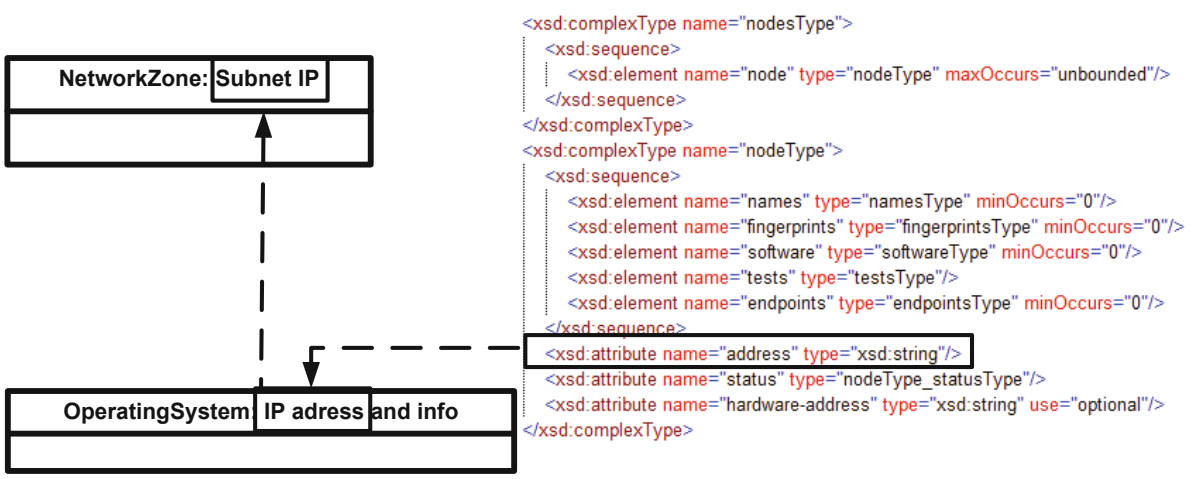

Fig. 5. Mapping of NetworkZone to NeXpose XML

Installation (e.g. Service or OS) - a common computer network could include 30 systems which all use Windows XP SP2, but with different security patches installed. This would in CySeMoL correspond 30 instances of the entity OperatingSystem, all related to a single SoftwareProduct (Windows XP SP2). NeXpose: All general information needed by CySeMoL to generate the entity.

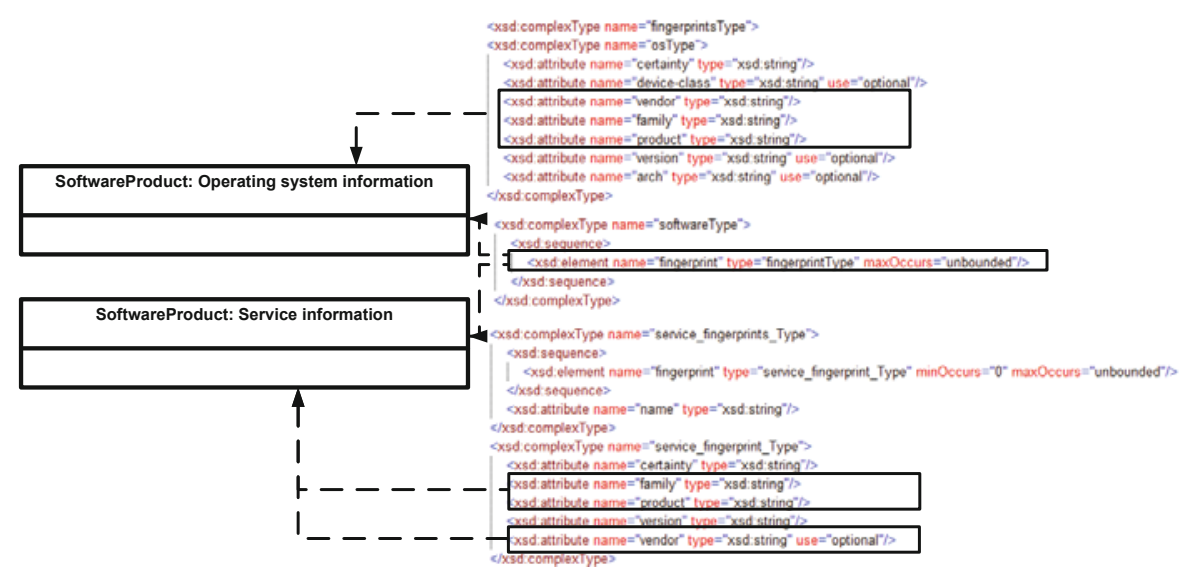

Fig. 6. Mapping of SoftwareProduct to NeXpose XML

\section{Example}

In this section we describe how we tested the implementation on a real network. We give a brief introduction to the background of the collected data. Afterwards we depict how the resulting auto-generated model looks like. 


\subsection{The Setup}

The main experimental setup was designed by the Swedish Defence Research Agency (FOI), with the support of the Swedish National Defence College (SNDC). Also, a group of computer security specialists and computer security researchers originating from various European governments, military, private sectors and academic institutions were part of designing the network architecture.

The environment was set to describe a simplified critical information infrastructure at a small electrical power utility. The environment was composed of 20 physical PC servers running a total of 28 virtual machines, divided into four VLAN segments. Various operating systems and versions thereof were used in the network, e.g. Windows XP SP2, Debian 5.0 and Windows Server 2003 SP1. Each host had several different network services operating, e.g. web-, mail-, media-, remote connection- and file sharing services. Furthermore, every host was more or less vulnerable through software flaws and/or poor configurations.

\subsection{The Result}

We performed an authenticated NeXpose scan on the setup environment and thereafter applied the mapping presented in chapter 4. The auto generated model consists of four instances of CySeMol's NetworkZone and 28 instances of CySeMol's OperatingSystem class. Furthermore 225 instances of the Service class and 141 instantiations of the SoftwareProduct class were automatically generated. The components were related based on the relations of CySeMol.

Figure 17, 8, and 9] show subsets of the resulting model, exemplary for one computer of the environment as the full model is too large to be shown here. In figure 7 the network zone that the computer belongs to is shown, followed by a consideration of the software and services that can be found on that machine. Finally figure 9] shows the computer and some of its attributes according to CySeMol, as well as the evidence that was set automatically.

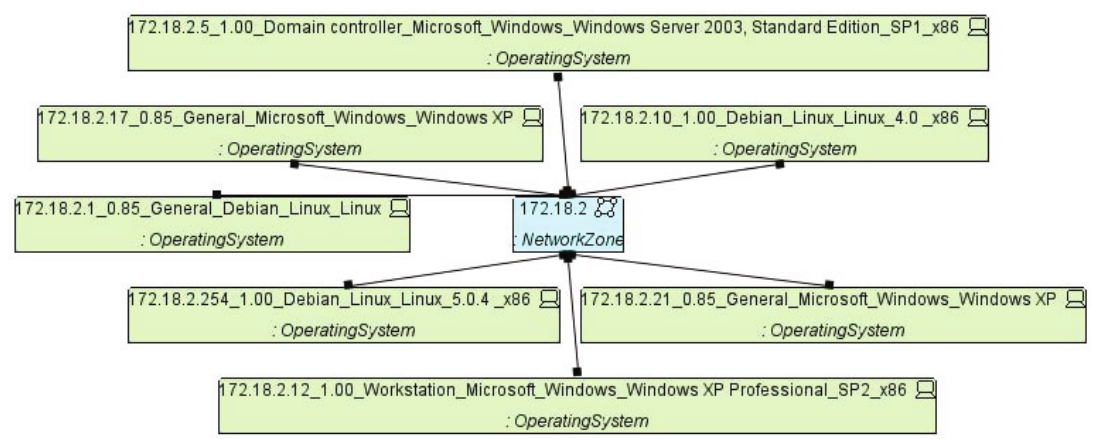

Format: Address_Certainty_Software-class_Vendor_Family_Product_Version

Fig. 7. Resulting model of one network zone 


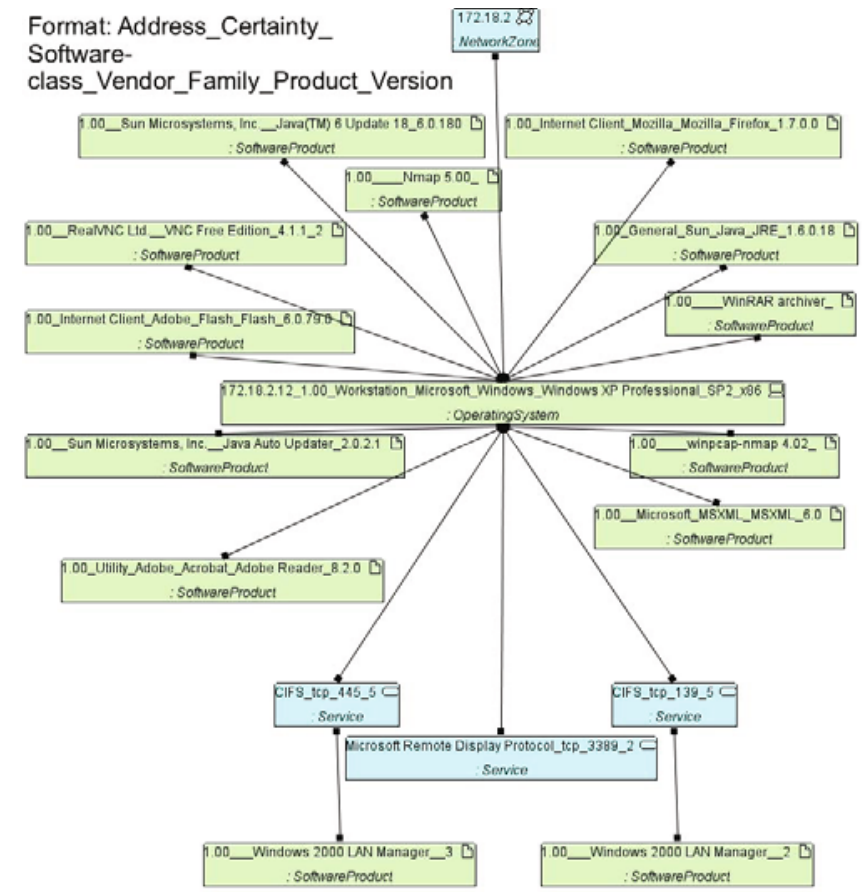

Fig. 8. One selected node

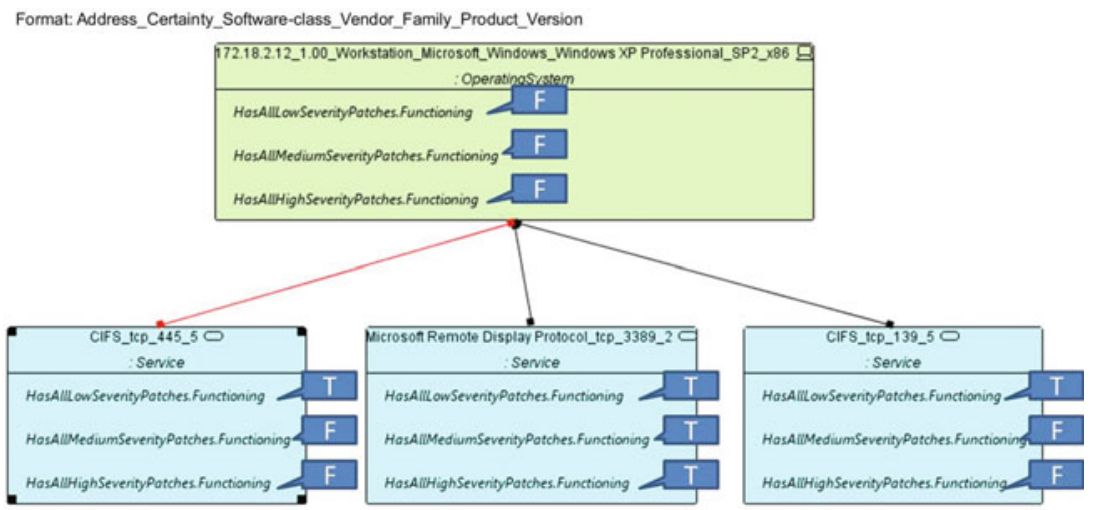

Fig. 9. The considered attributes of the node

\section{Discussion and Future Work}

This paper shows that vulnerability scanners can provide support for the creation of EA models. As mentioned earlier, scanners do not deliver complete EA models, but require some completion work. Their application however significantly reduces modeling effort and creates a model stub that can be complemented. 
The validity and reliability of the approach can be discussed from two different viewpoints: i) how much of the meta-model that can be captured, both in scope (i.e. how much of the meta-model that can be instantiated) and context (i.e. if the scanner provides all the information needed to accurately capture the context of a variable), and ii) how accurate a vulnerability scanner is at assessing the instantiated variables. Regarding i), most of the more modeling intensive concepts of CySeMoL are captured and all context are accurate. That is, the scanner provides e.g. all the information regarding vulnerabilities that $\mathrm{CySeMoL}$ requires. Regarding ii), the scanning accuracy in terms of assessing vulnerabilities is studied in [10]. The accuracy in terms of assessing software, operating systems and such is something that will be examined in future works.

It would also be interesting to look at other variables provided by automated vulnerability scanning, e.g. user accounts of systems and software clients (such as Adobe Reader). Furthermore, automated scanning could be mapped to more commonly used EA frameworks such as 12 to increase the usage of the method.

Additionally in future work it might be investigated how other data sources can be used to further reduce the manual tasks. Examples of such sources are access control lists, ERP systems, and UDDI registries. Especially how automatic data collection for the domains that so far not have been considered (the Business Layer and the information architecture) can be done, needs to be investigated. The goal is to minimize the manual effort to generate EA models.

Enterprises are changing permanently. Periodic scans leading to an automatic model update might therefore be implemented in the present tool as well.

It is also possible to collect information on vulnerabilities of services and software. This is something that we aim to incorporate in a future project.

\section{Conclusion}

In this paper we presented an extension of our previously developed tool that allows the automatic generation of elements for EA models. The input is provided by a vulnerability scanner, which was used to identify elements that were part of a computer network. Our implementation is generic even though CySeMol, a meta-model for security analysis, was used as a running example. The data gained from the vulnerability scanner can be used to instantiate any meta-model, once a mapping has been defined. The scan with NeXpose took less than an hour and the creation of the EA model using that data was next to instantaneous. Thus, it should be a viable option for EA architects. We have also illustrated the architecture of our implementation and described used components in detail. Finally, we have presented a practical application based on real data of our implementation. Thereby we have shown the feasibility of our approach.

\section{References}

1. Aier, S., Buckl, S., Franke, U., Gleichauf, B., Johnson, P., Närman, P., Schweda, C., Ullberg, J.: A survival analysis of application life spans based on enterprise architecture models. In: 3rd International Workshop on Enterprise Modelling and Information Systems Architectures, Ulm, Germany, pp. 141-154 (2009) 
2. Aier, S., Kurpjuweit, S., Saat, J., Winter, R.: Enterprise Architecture Design as an Engineering Discipline. AIS Transactions on Enterprise Systems 1(1), 36-43 (2009)

3. BiZZdesign: BiZZdesign Architect (March 2011) http://www.bizzdesign.com

4. Breu, R.: Ten principles for living models - a manifesto of change-driven software engineering. In: International Conference on Complex, Intelligent and Software Intensive Systems, pp. 1-8 (2010)

5. Buckl, S., Matthes, F., Neubert, C., Schweda, C.M.: A wiki-based approach to enterprise architecture documentation and analysis. In: 17th European Conference on Information Systems, pp. 1-13 (2009)

6. Buschle, M., Ullberg, J., Franke, U., Lagerström, R., Sommestad, T.: A Tool for Enterprise Architecture Analysis Using the PRM Formalism. In: Soffer, P., Proper, E. (eds.) CAiSE Forum 2010. LNBIP, vol. 72, pp. 108-121. Springer, Heidelberg (2011)

7. Fischer, R., Aier, S., Winter, R.: A federated approach to enterprise architecture model maintenance. Enterprise Modelling and Information Systems Architectures 2(2), 14-22 (2007)

8. Friedman, N., Getoor, L., Koller, D., Pfeffer, A.: Learning probabilistic relational models. In: Proc. of the 16th International Joint Conference on Artificial Intelligence, pp. 1300-1309. Morgan Kaufmann (1999)

9. Hafner, M., Winter, R.: Processes for enterprise application architecture management. In: Proceedings of the 41st Hawaii International Conference on System Sciences, pp. 396-406 (2008)

10. Holm, H., Sommestad, T., Almroth, J., Persson, M.: A quantitative evaluation of vulnerability scanning. Information Management \& Computer Security 19(4), 231-247 (2011)

11. Johnson, C., Quinn, S., Scarfone, K., Waltermire, D.: The technical specification for the security content automation protocol (SCAP). NIST Special Publication 800, 126 (2009)

12. Lankhorst, M.M.: Enterprise Architecture at Work: Modelling, Communication and Analysis, 2nd edn. Springer, Heidelberg (2009)

13. Mell, P., Scarfone, K., Romanosky, S.: A complete guide to the common vulnerability scoring system version 2.0. In: Published by FIRST-Forum of Incident Response and Security Teams (2007)

14. Rapid7: NeXpose (March 2011), http://www.rapid7.com/

15. Software AG: ARIS for SAP (2011), http://www.softwareag.com/corporate/ products/aris_platform/aris_implementation/aris_sap

16. Sommestad, T., Ekstedt, M., Johnson, P.: A probabilistic relational model for security risk analysis. Computers \& Security 29(6), 659-679 (2010)

17. Sommestad, T., Ekstedt, M., Nordström, L.: A case study applying the Cyber Security Modeling Language (2010)

18. Sousa, P., Lima, J., Sampaio, A., Pereira, C.: An Approach for Creating and Managing Enterprise Blueprints: A Case for IT Blueprints. In: Albani, A., Barjis, J., Dietz, J.L.G. (eds.) CIAO! 2009. LNBIP, vol. 34, pp. 70-84. Springer, Heidelberg (2009)

19. The Open Group: The Open Group Architecture Framework (TOGAF) - version 9. The Open Group (2009)

20. Troux Technologies: Metis (March 2011), http://www.troux.com/products/

21. Zachman, J.A.: A framework for information systems architecture. IBM Systems Journal 26, 276-292 (1987) 